\title{
ANALYTIC FUNCTIONS AND JORDAN ARCS
}

\section{LAWRENCE ZALCMAN}

Let $J$ be a Jordan arc lying on the complex sphere $S$, and let $F: S \rightarrow S$ be a homeomorphism. In several different connections (see e.g., [4]) it has been asked if the conformality of $F$ off $J$ implies that $F$ is a linear fractional transformation. The purpose of this brief note is to point out that a recent example of Rothberger [5] can be used to answer this question in the negative.

Rothberger proves the

THEOREM. There exists a function $F: S \rightarrow S$ such that

(1) $F$ is an autohomeomorphism of $S$;

(2) $F$ is conformal off a totally disconnected perfect subset $E$ of $S \backslash\{\infty\}$;

(3) $F$ is not a linear fractional transformation.

By a theorem of Antoine [1, p. 302], $E$ lies on a simple Jordan arc $J$. Taking this $J$ and the $F$ of the theorem, we obtain the required example.

A second, less explicit construction also deserves mention. Suppose there exists a (nonconstant) continuous function $f$ on $S$, bounded and analytic on $S \backslash J$, which satisfies a Lipschitz condition

$$
|f(z)-f(w)|<M|z-w|, \quad z, w \in S \backslash\{\infty\},
$$

$M$ an absolute constant. Then it is easy to see that the function $F(z)=M z+f(z)$ is a nontrivial autohomeomorphism of $S$ which is conformal off $J$. The interesting question of necessary and sufficient conditions (on $J$ ) for the existence of such an $f$ seems to be open; related problems have been studied by Carleson [2] and Dolzhenko [3].

\section{REFERENCES}

1. L. Antoine, Sur l'homéomorphie de deux figures et leurs voisinages, J. Math. Pures Appl. (8) 4 (1921), 221-325.

2. L. Carleson, On null-sets for continuous analytic functions, Ark. Mat. 1 (1950), 311-318.

3. E. P. Dolzhenko, The removability of singularities of analytic functions, Uspehi Mat. Nauk (112) 18 (1963), 135-142. (Russian)

4. F. Gardiner and I. Kra, On the boundary of Teichmuller spaces, (to appear).

5. F. Rothberger, On a conformal mapping problem of Stoilow and of Wolibner, Colloq. Math. 17 (1967), 61-69.

Massachusetts Institute of Technology

Received by the editors September 18, 1967. 